\title{
Lupus eritematoso discóide na infância
}

\author{
Discoid lupus erythematosus in childhood
}

Maria Carolina de A. Sampaio', Clovis Artur A. Silva ${ }^{2}$, Rosa Maria R. Pereira ${ }^{3}$, Zilda Najjar P. de Oliveira ${ }^{4}$

\section{RESUMO}

Objetivo: Realizar revisão da literatura sobre o lupus eritematoso discóide (LED) na infância.

Fontes de dados: Livros-texto e artigos de revistas indexadas pelo Medline e SciELO nos últimos dez anos, usando as seguintes palavras-chave: "discoid lupus erythematosus", "chronic cutaneous lupus erythematosus", "lupus erythematosus in childhood", "lupus erythematosus in children", "discoid lupus erythematosus in childhood", "discoid lupus erythematosus in children".

Síntese dos dados: A idade de início da doença ocorre predominantemente entre cinco e dez anos e a história familiar de lupus eritematoso está presente em 11 a $35 \%$ dos casos. A relação gênero feminino/masculino varia de $1 / 1$ a 2,4/1. Por sua vez, 24 a $27 \%$ dos pacientes com LED desenvolvem lupus eritematoso sistêmico (LES). Lesões discóides localizadas (que acometem cabeça e pescoço) são observadas em 56 a $75 \%$ dos pacientes. A face é o local mais acometido. O LED localizado e o generalizado apresentam evolução semelhante. Os achados histológicos são típicos, mostrando dermatite de interface. IgM e IgG são os depósitos mais freqüentes na zona da membrana basal da epiderme. Os tratamentos geralmente utilizados são: fotoproteção, corticosteróides tópicos e antimaláricos. Imunossupressores, talidomida, dapsona e retinóides podem ser usados nos casos refratários.

Conclusões: O LED da infância parece ter pequeno predomínio no gênero feminino, alta prevalência de história familiar de lupus eritematoso e elevada proporção que evolui para a forma sistêmica da doença, comparada ao LED do adulto. O LED localizado e o generalizado apresentam prognósticos semelhante. Os achados histológicos não foram diferentes daqueles descritos no LED do adulto.

Palavras-chave: lupus eritematoso discóide; lupus eritematoso sistêmico; vasculite.

\section{ABSTRACT}

Objective: To review the literature about discoid lupus erythematosus (DLE) in childhood.

Data sources: Textbooks and journals indexed for Medline and SciELO in the last ten years. The following key-words were searched: "discoid lupus erythematosus", "chronic cutaneous lupus erythematosus", "lupus erythematosus in childhood", "lupus erythematosus in children", "discoid lupus erythematosus in childhood", "discoid lupus erythematosus in children”.

Data synthesis: Disease onset occurs predominantly between five and ten years old. Family history of lupus erythematosus is present in $11-35 \%$ of cases. The female/ male ratio varies from $1 / 1$ to $2.4 / 1$. Development of systemic lupus erythematosus (SLE) is present in $24-27 \%$ of patients. Localized discoid lesions are observed in $56-75 \%$ of patients. The face is the most frequent site of involvement. Localized and generalized DLE have a similar course. The histological findings are typical, with interface dermatitis. IgM and IgG are the most frequent deposits in the basement membrane zone. The treatments usually used are: sunscreens, topical corticosteroids and antimalarials. Immunosuppressive agents, thalidomide, dapsone and retinoids can be used in refractory cases.

Conclusions: Childhood DLE shows slight female predominance, high prevalence of familiar history of lupus erythematosus, and it evolves to systemic lupus erythematosus in a higher proportion than adult DLE. Disseminated DLE and localized DLE have similar prognosis. The histological findings did not differ from those described in adults.

Key-words: lupus erythematosus, discoid; lupus erythematosus, systemic; vasculitis.

\footnotetext{
${ }^{1}$ Mestranda em Ciências do Departamento de Dermatologia da Faculdade de Medicina da Universidade de São Paulo (FMUSP)

${ }^{2}$ Professor colaborador e doutor do Departamento de Pediatria da FMUSP, responsável pela Unidade de Reumatologia Pediátrica do Departamento de Pediatra da FMUSP

${ }^{3}$ Professora livre docente da Disciplina de Reumatologia da FMUSP ${ }^{4}$ Professora doutora do Departamento de Dermatologia da FMUSP

Endereço para correspondência: Maria Carolina de A. Sampaio Rua Bergamota, 388, apto. 33 CEP 05468-000 - São Paulo/SP E-mail: mcasampaio@yahoo.com

Recebido em: 6/12/2006 Aprovado em: 26/2/2007
} 


\section{Introdução}

O lupus eritematoso discóide (LED) é raro na infância e adolescência, sendo pouco estudado nesta faixa etária ${ }^{(1,2)}$. Os trabalhos da literatura mostram que existem algumas peculiaridades dessa entidade em crianças e adolescentes, em relação aos adultos ${ }^{(1,3,4-6)}$. Na prática clínica, o aspecto mais preocupante é a maior tendência do LED para evoluir para lupus eritematoso sistêmico juvenil (LESJ) em pacientes com idade abaixo de 18 anos.

\section{Revisão da literatura}

O lupus eritematoso é uma doença do tecido conectivo de natureza auto-imune, de etiologia multifatorial e caráter espectral. Apresenta uma variedade de sintomas e sinais clínicos, desde manifestações cutâneas exclusivas até um quadro sistêmico grave e fatal ${ }^{(7)}$. Acomete principalmente mulheres jovens, sendo que aproximadamente $15 \%$ dos casos de lupus eritematoso sistêmico (LES) se iniciam antes dos 16 anos $^{(3)}$ e, por volta de $3 \%$ dos casos de LED, antes dos dez anos de idade ${ }^{(1)}$.

Existem diversas manifestações cutâneas do lupus eritematoso. Estas podem ser divididas em específicas e inespecíficas, de acordo com suas características clínicas e histológicas. As lesões cutâneas específicas do lupus eritematoso são classificadas como: lesão aguda, subaguda e crônica. Tal terminologia também reflete a duração e a gravidade do quadro ${ }^{(8)}$. Estas lesões ocorrem exclusivamente no lupus eritematoso, diferentemente das lesões inespecíficas, que são encontradas também em outras doenças. A lesão aguda corresponde ao eritema macular ou a pápulas e placas eritematosas na região malar, que, ocasionalmente, podem se generalizar (Figura 1 e 2). A lesão subaguda corresponde a placas eritematosas e descamativas (psoriasiformes) ou anulares, com marcada fotossensibilidade.

O lupus eritematoso cutâneo crônico, por sua vez, apresenta como quadro clássico o LED. Este ocorre nas áreas fotoexpostas, principalmente na face, couro cabeludo, orelhas, porção anterior do tórax e superfície de extensão dos membros superiores. É caracterizado por placas ovaladas, eritematosas, infiltradas, podendo apresentar escama aderente com espícula córnea folicular, discromia, telangiectasia, alopecia e atrofia (Figura 3).

$\mathrm{Na}$ infância, o lupus eritematoso cutâneo crônico é pouco freqüente ${ }^{(4,5)}$. Menos de 3\% dos casos de LED iniciam-se antes dos dez anos de idade ${ }^{(1)}$. Tebbe e Orfanos ${ }^{(2)}$ mostram que 3,1\% dos casos de LED têm início antes dos 20 anos e Cherif et a $l^{(1)}$ encontram 2,7\% dos casos de LED com início em crianças abaixo de dez anos. Moises-Alfaro et al ${ }^{(5)}$ observaram, no Instituto Nacional de Pediatria da Cidade do México, um caso de LED para cada 18 casos de LES.

$\mathrm{Na}$ faixa etária pediátrica, a média da idade de início do quadro varia de cinco a dez anos e a presença de história familiar de lupus eritematoso é encontrada em 11 a $35 \%$ dos $\operatorname{casos}^{(4,5)}$. Entretanto, no adulto com LED, a história familiar de lupus eritematoso é menos freqüente, ocorrendo em 1 a $4,4 \%$ dos $\operatorname{casos}^{(9)}$.

$\mathrm{Na}$ criança, parece haver menor predomínio do gênero feminino e alguns autores não encontrem predomínio neste gênero ${ }^{(1,4,6)}$. Moises-Alfaro et a ${ }^{(5)}$ mostram predomínio no gênero feminino de 2,4/1 na casuística por eles analisada. Já naquelas com menos de dez anos de idade, a relação entre gênero feminino e masculino foi de $5 / 1$.

Vários autores ressaltam que crianças e adolescentes com LED mostram maior risco de evolução para a forma sistêmica, visto que 24 a $27 \%$ das crianças com LED desenvolvem $\operatorname{LES}^{(4,5)}$. Outro aspecto relevante é que, na infância e adolescência, parece não haver diferença de prognóstico entre o LED localizado (que acomete cabeça e pescoço) e o LED generalizado (que acomete também outros segmentos) $)^{(5,6)}$. Este achado difere dos adultos com LED, pois, nos últimos, pacientes com envolvimento cutâneo mais extenso apresentam maior freqüência de sintomas sistêmicos ${ }^{(10)}$. Por sua vez, George e Tunnessen ${ }^{(6)}$ sugerem que as crianças com LED e que evoluem para LES podem evoluir com doença sistêmica grave, nefrite, comprometimento do sistema nervoso central, cardite, pleurite, vasculite e até óbito.

A área mais acometida no LED da infância é a face (Figuras 1, 2 e 3), seguindo-se o acometimento das orelhas, couro cabeludo, membros superiores e tronco, segundo Van Gysel et $a l^{(4)}$. O acometimento do tórax, couro cabeludo, orelhas e região pré-tibial é evidenciado no estudo de Moises-Alfaro et al ${ }^{(5)}$. O LED localizado (que acomete cabeça e pescoço) é descrito em $56 \%$ a $75 \%$ das crianças e adolescentes ${ }^{(1,4,5)}$. Lesões lineares seguindo as linhas de Blaschko (linhas observadas no mosaicismo cutâneo e que provavelmente refletem o trajeto das células ectodérmicas durante a embriogênese) também foram descritas no LED infantil ${ }^{(11,12)}$. O LED associado ao lupus eritematoso profundo foi pouco descrito na infância ${ }^{(13-16)}$. Um aspecto interessante evidenciado no LED da infância é a presença de fotossensibilidade, relatada em $30-80 \%$ dos $\operatorname{casos}^{(1,4,6)}$. 


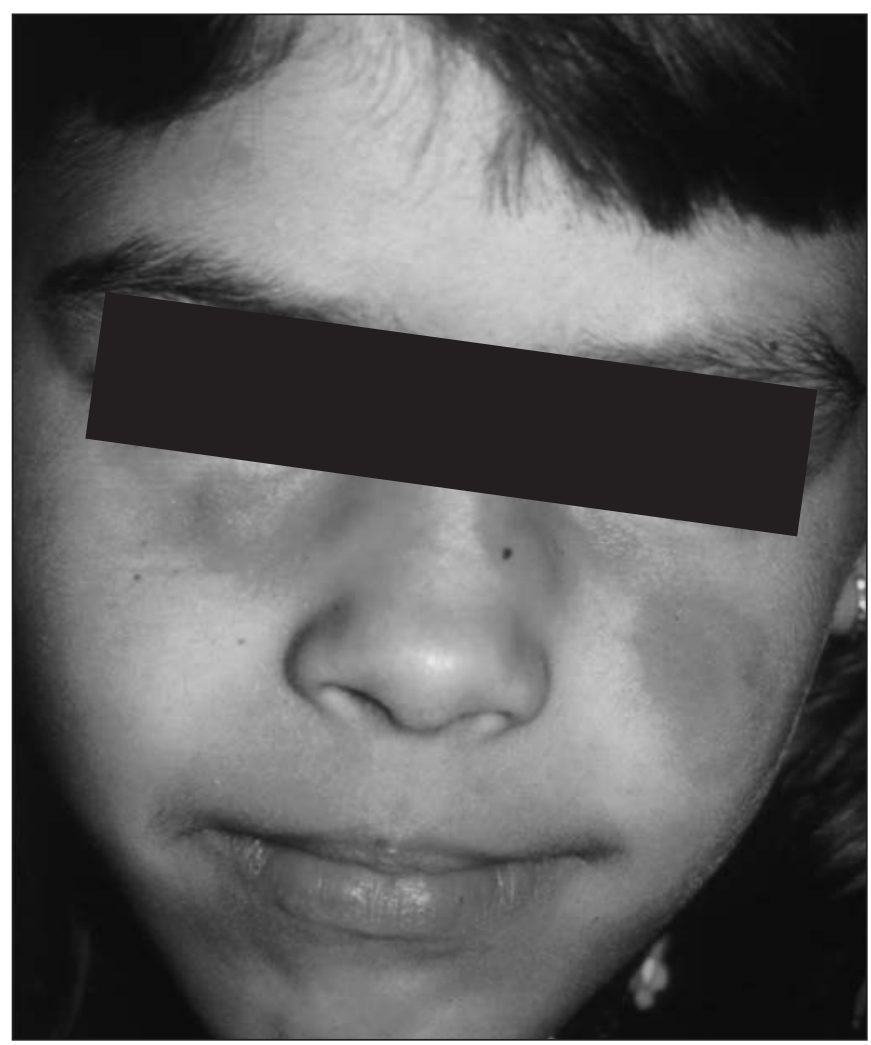

Figura 1 - Placas ovaladas, eritematosas, infiltradas

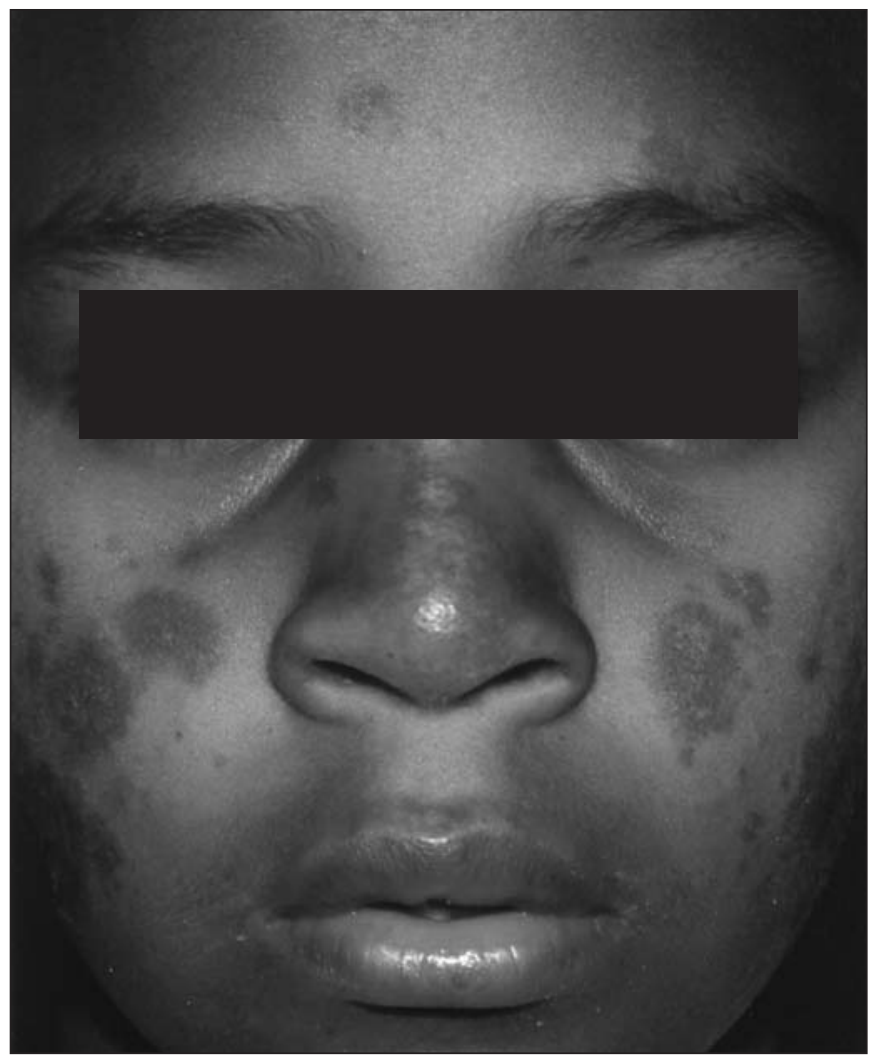

Figura 2 - Placas ovaladas hipercrômicas com centro eritêmatodescamativo e episclerite
O comprometimento de mucosas e semi-mucosas é pouco observado na faixa etária pediátrica e pode passar despercebido, pois, habitualmente, é assintomático. Van Gysel et al ${ }^{(4)}$ descreveram uma criança de dois anos com ulcerações orais e Cherif et al ${ }^{(1)}$ descreveram cinco casos de queilite do lábio inferior em 16 crianças com LED. Piras e Cottoni ${ }^{(17)}$ relataram um paciente com placas eritematosas hiperqueratóticas no palato duro. Por sua vez, no adulto, o acometimento de mucosas é mais freqüente e ocorre em 16 a $27 \%$ dos $\operatorname{casos}^{(9,18,19)}$.

Com relação aos auto-anticorpos séricos, não existe um marcador específico para o diagnóstico de LED. Entretanto, a presença de fator anti-nuclear (FAN) ocorre entre 26 e $63 \%$ dos $\operatorname{casos}^{(1,4,5)}$. Moisés-Alfaro et al ${ }^{(5)}$ evidenciaram positividade de outros auto-anticorpos: anti-DNA hélice simples em $22 \%$, anti-ENA em $33 \%$ e anti-Ro em 20\%. Entretanto, vale ressaltar que, nos pacientes com LED, a presença do anticorpo anti-DNA de dupla hélice e/ou anti-Sm sugere fortemente o diagnóstico de LES, cujo diagnóstico pode ser evolutivo, com o preenchimento de quatro dos 11 critérios de classificação diagnóstica do LES estabelecido pelo American College of Rheumatology.

O diagnóstico de LED pode ser confirmado pela histologia cutânea, embora esta tenha sido pouco estudada na infância e adolescência. Os achados histológicos encontrados por Moises-Alfaro et $a l^{(5)}$ e George et al ${ }^{(20)}$ foram semelhantes aos já descritos no adulto: graus variados de hiperqueratose, rolha córnea folicular, atrofia da epiderme, vacuolização das células da camada basal da epiderme e infiltrado inflamatório de células mononucleadas ao redor de vasos e anexos na derme.

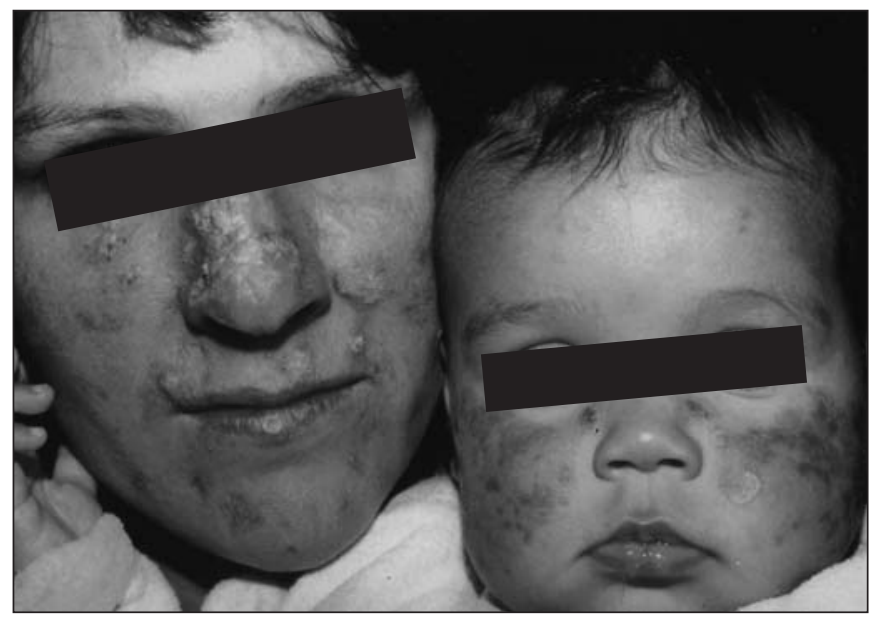

Figura 3 - Mãe com placas eritematosas, infiltradas, com superfície hiperqueratótica e lesões residuais atróficas e hipercrômicas. Filha com algumas placas eritematosas infiltradas e máculas hipercrômicas residuais na região malar 
Na imunofluorescência direta, a IgG foi o imuno-depósito encontrado na zona da membrana basal em cinco dos nove casos estudados por Moises-Alfaro et al ${ }^{(5)}$. Da mesma maneira, a imunofluorescência direta foi positiva em quatro dos cinco casos relatados por George et al ${ }^{(20)}$, sendo $\operatorname{IgM}$ e IgG os imunodepósitos mais freqüentes. No adulto, a $\operatorname{IgM}$ parece ser o depósito mais freqüente, embora pouco específico ${ }^{(21)}$.

Para o tratamento do LED, utilizam-se fotoprotetores, corticosteróide tópico, intralesional e sistêmico, antimaláricos, talidomida e dapsona ${ }^{(1,4,5)}$. Não há estudos controlados sobre o tratamento do LED na infância, sendo que a terapêutica atual baseia-se na experiência de especialistas e na terapêutica usada no adulto. Moisés-Alfaro $e t a^{(5)}$ encontraram resposta satisfatória em $59 \%$ dos casos. Os antimaláricos apresentam, na criança, perfil de segurança semelhante ao do adulto. Tais drogas são fotoprotetoras e permitem diminuir as doses prescritas dos corticosteróides, além de reduzirem as hiperlipidemias ${ }^{(22)}$.

O tratamento do LED visa melhorar aparência do paciente e evitar o desenvolvimento de cicatrizes e discromias. Os fotoprotetores e as medidas de proteção solar, tais como uso de roupas e chapéus, são essenciais. Os fotoprotetores precisam ser aplicados diariamente nas áreas fotoexpostas, devem ser de amplo espectro e com alto fator de proteção solar, de preferência acima de 15. Recomenda-se que o paciente os aplique logo pela manhã e os reaplique a cada duas horas, ou após contato com água ou sudorese intensa, mesmo em dias nublados. Os corticosteróides tópicos são muito utilizados, sendo sua potência escolhida de acordo com o local e características da lesão. Eles podem ser aplicados uma a duas vezes ao dia e, quando necessário, usá-los por tempo prolongado, devendo-se intercalar intervalos curtos (alguns dias) sem a medicação para evitar seus efeitos adversos locais, como a atrofia cutânea. Nas lesões da face são usados corticosteróides de baixa e média potência; nas lesões do tronco e membros podem ser utilizados os de média potência. Os corticosteróides de alta potência são usados nas lesões das plantas, palmas e nas lesões hipertróficas. Os corticosteróides intralesionais podem ser aplicados em lesões discóides crônicas refratárias aos corticosteróides tópico. Outros agentes de uso tópico como os retinóides, pimecrolimus e tacrolimus podem ser usados no LED, embora ainda pouco estudados ${ }^{(10,23)}$.

A terapia sistêmica está indicada quando as lesões não são controladas com as medicações tópicas ou quando há várias lesões concomitantes. Os antimaláricos são drogas muito utilizadas no LED. Estes, além de fotoprotetores, permitem reduzir as doses prescritas dos corticosteróides orais, diminuem os anticorpos antifosfolípides associados às tromboses auto-imunes e os lipídeos: LDL e triglicérides. Tais ações são importantes, principalmente no LED associado ao LES. A dose de sulfato de hidroxicloroquina ou difosfato de cloroquina é de aproximadamente $5 \mathrm{mg} / \mathrm{kg} / \mathrm{dia}$. Alguns autores recomendam que a dose de cloroquina em crianças não ultrapasse $3,5 \mathrm{mg} / \mathrm{kg} /$ dia para evitar a toxicidade oftalmológica ${ }^{(24)}$. Seus eventos adversos mais comuns são náusea e vômito ${ }^{(24)}$. Devido à toxicidade oftalmológica, deve-se fazer um exame oftalmológico a cada três a seis meses. Nos pacientes refratários, pode-se usar corticosteróide sistêmico, dapsona, talidomida, metotrexato, azatioprina, retinóides, entre outros ${ }^{(10,23)}$. Alguns autores empregam corticosteróides sistêmicos por curtos períodos na dose de 0,5 a $1 \mathrm{mg} / \mathrm{kg} / \mathrm{dia}$, juntamente com os antimaláricos, que têm início de ação demorado. Porém, acredita-se que os corticosteróides sistêmicos em doses baixas são pouco efetivos para o $\operatorname{LED}^{(23)}$. De preferência, a talidomida deve ser evitada em adolescentes e adultos jovens do gênero feminino pelo seu potencial teratogênico.

\section{Conclusões}

O LED é pouco freqüente na infância. Nesta faixa etária, parece haver pequeno predomínio de sua prevalência no gênero feminino e maior freqüência de evolução para a forma sistêmica. O LED localizado e o generalizado parecem apresentar prognósticos semelhantes. Por sua vez, a história familiar de lupus eritematoso é mais freqüente no LED com início na faixa pediátrica. $\mathrm{O}$ acometimento de mucosas é raro e os achados histológicos evidenciam dermatite de interface típica do lupus eritematoso. O tratamento do LED é geralmente realizado com fotoprotetores, corticosteróides tópicos e antimaláricos. 


\section{Referências bibliográficas}

1. Cherif F, Mebazaa A, Mokni M, El Euch D, Azaiz MI, Dhahri AB. Childhood discoid lupus erythematosus: a Tunisian retrospective study of 16 cases. Pediatr Dermatol 2003;20:295-8.

2. Tebbe B, Orfanos CE. Epidemiology and socioeconomic impact of skin disease in lupus erythematosus. Lupus 1997;6:96-104.

3. Tucker LB, Menon S, Schaller JG, Isenberg DA. Adult- and childhood-onset systemic lupus erythematosus: a comparison of onset, clinical features, serology, and outcome. Br J Rheumatol 1995;34:866-72.

4. Van Gysel D, de Waard-van der Spek FB, Oranje AP. Childhood discoid lupus erythematosus: report of five new cases and review of the literature. J Eur Acad Dermatol Venereol 2002;16:143-7.

5. Moises-Alfaro C, Berrón-Pérez R, Carrasco-Daza D, Gutiérrez-Castrellón P, Ruiz-Maldonado R. Discoid lupus erythematosus in children: clinical, histopathologic, and follow-up features in 27 cases. Pediatr Dermatol 2003;20:103-7.

6. George PM, Tunnessen WW Jr. Childhood discoid lupus erythematosus. Arch Dermatol 1993;129:613-7.

7. Sontheimer RD. Lupus erythematosus. In: Freedberg IM, Eisen AZ, Wolff K, Austen KF, Goldsmith LA, Katz SI, Fitzpatrick TB, editors. Dermatology in general medicine. $5^{\text {th }}$ ed. New York: McGraw-Hill; 1999. p. 1993-2009.

8. Sontheimer RD. The lexicon of cutaneous lupus erythematosus - a review and personal perspective on the nomenclature and classification of the cutaneous manifestations of lupus erythematosus. Lupus 1997;6:84-95.

9. Freitas THP, Proença NG. Lupus eritematoso cutâneo crônico: estudo de 290 pacientes. An Bras Dermatol 2003;78:703-12.

10. Callen JP. Collagen vascular diseases. J Am Acad Dermatol 2004;51: 427-39.

11. Green JJ, Baker DJ. Linear childhood discoid lupus erythematosus following the lines of Blaschko: a case report with review of the linear manifestations of lupus erythematosus. Pediatr Dermatol 1999;16:128-33.
12. Requena C, Torrelo A, de Prada I, Zambrano A. Linear childhood cutaneous lupus erythematosus following Blaschko lines. J Eur Acad Dermatol Venereol 2002;16:618-20.

13. Bachmeyer C, Aractingi S, Blanc F, Verola O, Dubertret L. Deep lupus erythematosus in children. Ann Dermatol Venereol 1992;119:535-41.

14. Nitta Y. Lupus erythematosus profundus associated with neonatal lupus erythematosus. Br J Dermatol 1997;136:112-4.

15. Muncaster A, Stewart G, Moss C, Southwood T. Facial lupus erythematosus profundus in a 9-year-old boy. J R Soc Med 1998;91:207-8.

16. Wimmershoff MB, Hohenleutner $U$, Landthaler M. Discoid lupus erythematosus and lupus profundus in childhood: a report of two cases. Pediatr Dermatol 2003;20:140-5

17. Piras D, Cottoni F. Mucosal involvement in childhood discoid lupus erythematosus. J Eur Acad Dermatol Venereol 2003;17:731-2.

18. Burge SM, Frith PA, Juniper RP, Wojnarowska F. Mucosal involvement in systemic and chronic cutaneous lupus erythematosus. $\mathrm{Br} \mathrm{J}$ Dermatol 1989;121:727-41.

19. Le Bozec P, La Guyadec T, Crickx B, Grossin M, Belaich S. Chronic lupus erythematosus in lupus disease. Retrospective study of 136 patients. Presse Med 1994;23:1598-602.

20. George PM, Hood AF, Rest EB. Histopathology and immunofluorescence of discoid lupus erythematosus in children. Pediatr Dermatol 1996;13:269-73.

21. Kontos AP, Jirsari M, Jacobsen G, Fivenson DP. Immunoglobulin M predominance in cutaneous lupus erythematosus. J Cutan Pathol 2005;32:352-5.

22. Meurer M. Childhood discoid lupus erythematosus and antimalarials. Dermatology 2003;207:133.

23. Callen JP. Update on the management of cutaneous lupus erythematosus. $\mathrm{Br}$ J Dermatol 2004;151:731-6.

24. Ziering CL, Rabinowitz LG, Esterly NB. Antimalarials for children: indications, toxicities, and guidelines. J Am Acad Dermatol 1993;28:764-70. 